\title{
Precursor T-cell lymphoblastic lymphoma extensively involving the mediastinum, pleura and pericardium: A case report
}

\author{
XIANHONG XIANG ${ }^{1}$, XIAOYAN WANG ${ }^{2}$, QINQIN YI ${ }^{2}$, LIN LIN $^{2}$, \\ XIANGSONG ZHANG ${ }^{2}$, HONG LIANG ${ }^{2}$ and JIANYONG YANG ${ }^{1}$ \\ Departments of ${ }^{1}$ Interventional Radiology and ${ }^{2}$ Nuclear Medicine, The First Affiliated Hospital, \\ Sun Yat-Sen University, Guangzhou, Guangdong 510080, P.R. China
}

Received January 16, 2014; Accepted June 26, 2014

DOI: $10.3892 / \mathrm{mco} .2014 .347$

\begin{abstract}
Precursor T-cell lymphoblastic lymphoma (T-LBL) is a rare type of malignant lymphoma, with clinical manifestations including diaphragmatic lymph node enlargement, accompanied by local oppression and/or systemic lymphoma symptoms. However, extensive involvement of the mediastinum, pleura and pericardium is rare in T-LBL cases. This is the case report of a T-LBL extensively involving the mediastinum, pleura and pericardium in a 54-year-old woman. The patient complained of anhelation, chest tightness and tiredness for $\sim 3$ months. A computed tomography (CT) scan of the chest revealed a diffuse mass of soft tissue density involving the mediastinum, pleura and pericardium. Several thoracocenteses indicated inflammatory changes and cytological examination of the pleural fluid and pleural biopsy under CT guidance identified no heterotypic cells. As ${ }^{18}$ F-fluorodeoxyglucose (FDG) positron emission tomography (PET)/CT imaging revealed a diffused moderate FDG uptake (maximum standard uptake value of 4) by the mediastinum, pleura and cardiac sac, we diagnosed a malignant lymphoma. We subsequently successfully performed needle biopsy under PET/CT guidance according to the PET/CT images and the diagnosis of T-LBL was pathologically confirmed.
\end{abstract}

\section{Introduction}

Lymphoma includes a set of malignant solid tumors originating from lymphatic hematopoietic tissues. The lesions are wide ranging, their pathological characteristics are variable, their classification is complex and imaging diagnosis is difficult.

Correspondence to: Professor Jianyong Yang, Department of Interventional Radiology, The First Affiliated Hospital, Sun Yat-Sen University, 58 Zhongshan 2nd Road, Guangzhou, Guangdong 510080, P.R. China

E-mail: med.interventional@163.com

Key words: precursor T-cell lymphoblastic lymphoma, mediastinum, pleura, pericardium, positron emission tomography/computed tomography
Precursor T-cell lymphoblastic lymphoma (T-LBL) is a rare type of malignant lymphoma, with manifestations including lymph node enlargement, hepatosplenomegaly and annular erythema (1). The nasal mucosa, tonsils, oral cavity, skin, small intestine, or mediastinum may be involved in T-LBL (2); however, extensive involvement of the mediastinum, pleura and pericardium is rare (3-6). This is the case report of a T-LBL extensively involving the mediastinum, pleura and pericardium in a 54-year-old woman. The patient was diagnosed with malignant lymphoma following positron emission tomography/computed tomography (PET/CT) imaging. A subseqent biopsy according to the PET/CT images confirmed the diagnosis of T-LBL.

\section{Case report}

The patient was a 54-year-old woman, complaining of anhelation, chest tightness and tiredness for $\sim 3$ months, without palpitations or chest pain. The patient had no history of tuberculosis, hepatitis, or other communicable diseases. Following admission, the biochemical tests revealed a serum potassium level of $2.93 \mathrm{mmol} / \mathrm{l}$, a blood chlorine level of $84 \mathrm{mmol} / \mathrm{l}$ and a serum albumin level of $30.6 \mathrm{~g} / \mathrm{l}$. In addition, the purified protein derivative-immunoglobulin $\mathrm{G}$ was negative and the concentration of cancer antigen 125 in the serum was $99.2 \mathrm{U} / \mathrm{ml}$. Color doppler echocardiography revealed a massive pericardial effusion and a chest CT scan revealed a diffuse mass of soft tissue density involving the mediastinum, accompanied by pleural thickening, pericardial thickening, pleural effusion and pericardial effusion. The mass surrounded the trachea and the blood vessels in the mediastinum. On contrast-enhanced $\mathrm{CT}$, the thickened pleural and pericardial mass was slightly to moderately enhanced. Several thoracocenteses indicated inflammatory changes and the cytological examination of the pleural fluid and conventional pleural biopsy under CT guidance (performed twice, tissue block size 3x8 mm and 3x10 mm, respectively) identified no heterotypic cells. Although on ${ }^{18} \mathrm{~F}$ fluorodeoxyglucose (FDG) PET/CT imaging the lesions exhibited a moderate FDG uptake, with a maximum standard uptake value of 4, a diagnosis of malignant lymphoma was considered. Based on the PET/CT images that showed an active site, a biopsy guided by PET/CT was performed and a pathological tissue sample of $\sim 3 \times 15 \mathrm{~mm}$ was removed. On 

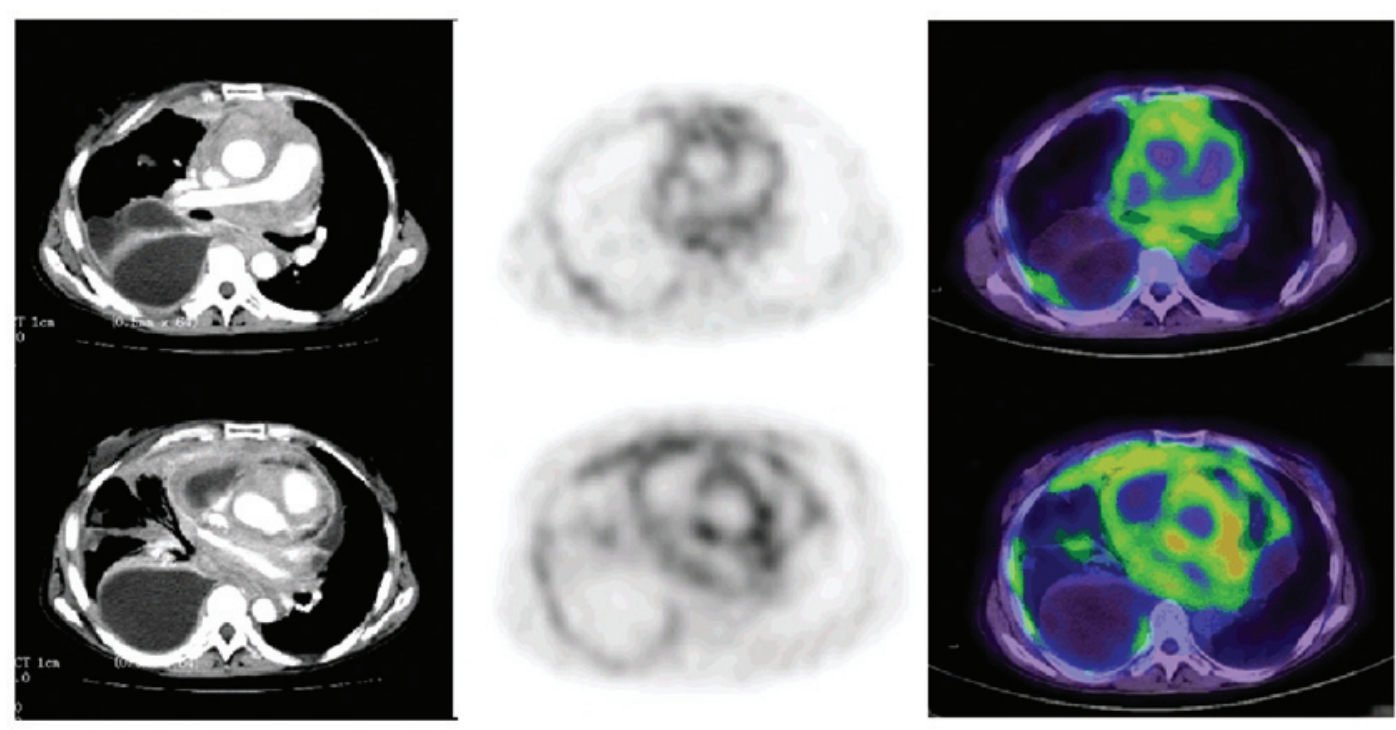

Figure 1. Contrast-enhanced computed tomography and positron emission tomography/computed tomography images revealing a diffuse mass in the mediastinum, pericardium and diaphragmatic pleura, which are extensively thickened and exhibit areas with increased metabolic activity.

microscopic examination (hematoxylin and eosin staining), the lesion was composed of numerous middle-sized lymphocytes with a diffuse distribution pattern. The immunohistochemical results were as follows: $\mathrm{CD}^{+}, \mathrm{CD}^{+}, \mathrm{CD}^{+}, \mathrm{CD}^{+}$, $\mathrm{TDT}^{+}$, $\mathrm{CD}^{2} 9^{+}, \mathrm{CD}^{+} 0^{+}, \mathrm{CD}^{+}, \mathrm{Ki}^{+}(\sim 80 \%), \mathrm{CD}^{-}, \mathrm{UCHL1}^{-}, \mathrm{TIA}^{-}$ Granzyme- ${ }^{-}, \mathrm{CK}^{-}, \mathrm{CK}_{19}{ }^{-}, \mathrm{CD} 20^{-}, \mathrm{CD}_{21}{ }^{-}$and $\mathrm{CD} 23^{-}$. The comprehensive hematoxylin and eosin stain morphology and the immunohistochemical results confirmed the diagnosis of T-LBL.

Oral and written consent was obtained from the patient prior to all the invasive interventions. Since all such interventions are for clinical routine inspection or treatment, the present study was exempt from medical ethics review.

\section{Discussion}

Lymph cell lymphoma was first described in detail in 1916 by Sternberg (http://reedsternbergcells.org/helpful-informationabout-reed-sternberg-cells). In 1973, Smith (7) reported that lymphoblastic lymphoma originated from thymus lymphocytes. Barcos and Lukes (8) described a 'nuclear lymphoid cell lymphoma', which was officially named 'lymph cell lymphoma' in 1975. Currently, the World Health Organization refers to the T-cell-derived lymphocytoblast lymphoma as 'T-LBL' (9).

T-LBL is a rare malignant lymphoma. The median age at onset for T-LBL is 16 years (range, 4-84 years). Children and adolescents are the most vulnerable, accounting for $~ 30-40 \%$ of all cases of T-LBL, with only $3-4 \%$ of the patients being adults. The male:female ratio is reported to be 2.5-3:1 (10). The typical clinical manifestation of diaphragmatic lymph node enlargement is accompanied by local oppression and systemic lymphoma symptoms. The tumor often grows quickly in the anterior mediastinum (50-80\%) and may infiltrate the central nervous system, the gonads and even the bone marrow. The clinical manifestations in our patient were mainly diffuse nodules in the mediastinum, pericardium and diaphragmatic pleura, bilateral pleural thickening and pleural and pericardial effusion. The patient was an adult female, aged 54 years and this disease exhibits a significantly lower incidence in this population; we identified no similar cases reported in relevant literature.

${ }^{18} \mathrm{~F}-\mathrm{FDG}$ imaging may provide an important basis for the diagnosis and differential diagnosis of malignant lymphoma. Malignant lymphoma is generally characterised by a higher cell density compared to other cancer cells and, thus, several lymphomas exhibit high FDG uptake (10). Following a review of several studies, Baba et al (1) concluded that the degree of FDG uptake is largely dependent on lymphoma histology, with the aggressive type usually exhibiting higher uptake. Compared to conventional CT, ${ }^{18} \mathrm{~F}-\mathrm{FDG}$ imaging exhibits higher sensitivity and specificity and may help prognosis and treatment.

A previous study by Reske (11) analyzed 15 studies on lymphoma, involving a total of 723 patients, and summarized the results of FDG PET imaging. The sensitivity of FDG PET imaging was reported to be $71-100 \%$, with a specificity of $69-100 \%$ and a negative predictive value of $80-100 \%$, whereas with CT, the specificity and positive predictive value were very low (4-31 and 19-60\%, respectively). Hernandez-Maraver et al (12) analyzed 47 cases of lymphoma patients diagnosed by PET/CT imaging and compared the results to those of PET or CT imaging alone. The results of PET/CT in 11 patients (10 with Non-Hodgkin's lymphoma and 1 with Hodgkin's lymphoma) revealed an increase in stage $(\mathrm{P}=0.012)$. The PET/CT imaging detection of nodal and extranodal lesions exhibited a significantly higher sensitivity compared to the sensitivity of CT or PET imaging performed separately. ${ }^{18} \mathrm{~F}-\mathrm{FDG}$ PET imaging may also help differentiate between lymphoma subtypes.

However, ${ }^{18} \mathrm{~F}$-FDG PET imaging in the diagnosis of lymphomas has certain limitations. T-cell lymphomas are complicated and the majority exhibit low to moderate FDG uptake. If the nidus is too small or of low-level malignancy, this examination may yield false-negative results. In addition, inflammation, granulomas, physiological uptake 

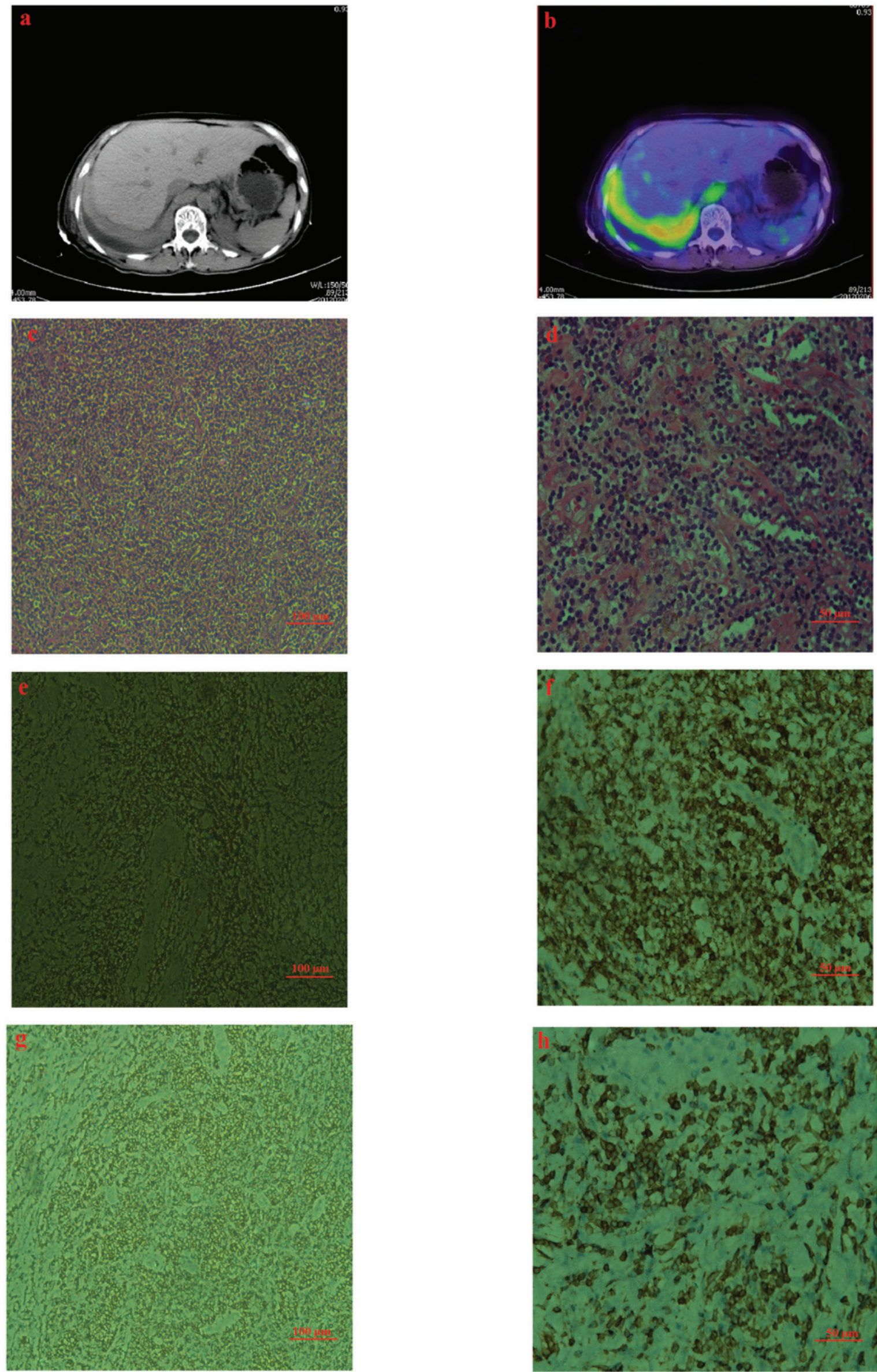

Figure 2. Chest (a) computed tomography and (b) positron emission tomography/computed tomography images; the latter shows the active lesions, which were subsequently biopsied. (c and d) The microscopic examination (hematoxylin and eosin staining) revealed a nodular distribution of lymphoid cells of medium size, exhibiting fine chromatin and some visible small nucleoli. (e-h) The immunohistochemical examination revealed CD2, CD3, CD5, CD7 and TDT positivity. 
by the gastrointestinal tract, urinary tract and muscle, as well as thymus and bone marrow proliferative response may be characterized as false-positives (13).

Although the patient had to undergo biopsy twice under conventional CT guidance to obtain sufficient specimens ( $3 \times 8 \mathrm{~mm}$ and $3 \times 10 \mathrm{~mm})$, we were unable to identify tumor cells. Based on the PET/CT images that showed an active site, a biopsy guided by PET/CT was performed and a pathological tissue sample of $\sim 3 \times 15 \mathrm{~mm}$ was removed. Therefore, ${ }^{18} \mathrm{~F}-\mathrm{FDG}$ $\mathrm{PET} / \mathrm{CT}$ imaging may also guide the selection of clinical puncture biopsy sites and improve the accuracy and consequent success rates of the procedure. Although histopathological assessment remains the gold standard for the diagnosis of T-cell lymphoma, there are anthropogenic limitations, as well as limitations regarding the characteristics of the lesions per se (e.g., necrosis and inflammation) inherent in puncture biopsies $(5,6)$. Therefore, the proper selection of the biopsy site and technique is crucial for accurate pathological diagnosis. In our patient, improper selection of the sampling position during the initial biopsy led to a misdiagnosis. Finally, with PET/CT imaging and PET/CT scan-guided puncture biopsy in the right posterior pleura led to the confirmation of the diagnosis of T-LBL. These results suggest that PET/CT scan may be clinically valuable in the selection of biopsy positions in T-LBL cases.

In conclusion, we presented a rare case of T-LBL extensively involving the mediastinum, pleura and pericardium and demonstrated that PET/CT may be of significant value in the clinical diagnosis of lymphoma and may be used as guidance for the selection of puncture biopsy sites.

\section{References}

1. Baba S, Abe K, Isoda T, Maruoka Y, Sasaki M and Honda H: Impact of FDG-PET/CT in the management of lymphoma. Ann Nucl Med 25: 701-716, 2011.
2. Ko YH and Lee JD: Peripheral T-cell lymphomas - clinicopathologic and immunophenotypic analysis of 25 cases. J Korean Med Sci 6: 329-337, 1991.

3. Makis W, Novales-Diaz JA and Lisbona R: Cardiac T-cell lymphoma imaged with F-18 FDG PET-CT and correlative imaging. Clin Nucl Med 35: 332-334, 2010.

4. Lepeak LM, Yang DT and Chang JE: Extranodal NK/T-cell lymphoma presenting with primary cardiac involvement. Hematol Rep 3: e9, 2011.

5. Vega F, Padula A, Valbuena JR, Stancu M, Jones D and Medeiros LJ: Lymphomas involving the pleura: a clinicopathologic study of 34 cases diagnosed by pleural biopsy. Arch Pathol Lab Med 130: 1497-1502, 2006.

6. Patel J, Melly L and Sheppard MN: Primary cardiac lymphoma: B- and T-cell cases at a specialist UK centre. Ann Oncol 21: 1041-1045, 2010.

7. Wybran J and Fudenberg HH: Thymus-derived rosette-forming cells in various human disease states: cancer, lymphoma, bacterial and viral infections, and other diseases. J Clin Invest 52: 1026-1032, 1973.

8. Rilke F, Clemente C, Pilotti S and Musumeci R: Convoluted cell lymphoblastic lymphoma (author's transl). Tumori 61: 377-391, 1975 (In Italian).

9. Chan JK: The new World Health Organization classification of lymphomas: the past, the present and the future. Hematol Oncol 19: 129-150, 2001.

10. Goldsby RE and Carroll WL: The molecular biology of pediatric lymphomas. J Pediatr Hematol Oncol 20: 282-296, 1998.

11. Reske SN: PET and restaging of malignant lymphoma including residual masses and relapse. Eur J Nucl Med Mol Imaging 30 (Suppl 1): S89-S96, 2003.

12. Hernandez-Maraver D, Hernandez-Navarro F, Gomez-Leon N, Coya J, Rodriguez-Vigil B, Madero R, Pinilla I and Martin-Curto LM: Positron emission tomography/computed tomography: diagnostic accuracy in lymphoma. Br J Haematol 135: 293-302, 2006.

13. Castellucci P, Zinzani P, Pourdehnad M, et al: ${ }^{18} \mathrm{~F}-\mathrm{FDG}$ PET in malignant lymphoma: significance of positive findings. Eur J Nucl Med Mol Imaging 32: 749-756, 2005. 